\title{
Editorial: Atmospheric Electricity
}

\author{
Irina Mironova ${ }^{1,2 *}$, Martin Füllekrug ${ }^{3}$, Konstantinos Kourtidis $^{4}$ and Evgeny Mareev ${ }^{5}$ \\ ${ }^{1}$ Faculty of Physics, Saint Petersburg State University, Saint Petersburg, Russia, ${ }^{2}$ National Research Nuclear University MEPhl, \\ Moscow, Russia, ${ }^{3}$ Deptment of Electronic and Electrical Engineering, University of Bath, Bath, United Kingdom, ${ }^{4}$ Deptment of \\ Environmental Engineering, Democritus University of Thrace, Xanthi, Greece, ${ }^{5}$ Institute of Applied Physics, Russian Academy of \\ Sciences, Nizhny Novgorod, Russia
}

Keywords: atmospheric electricity, global electric circuit, magnetosphere-ionosphere-atmosphere, Schumann resonances, aerosol and cloud microphysics

\section{Editorial on the Research Topic}

\section{Atmospheric Electricity}

Atmospheric electricity is related to a broad range of disciplines, including the global atmospheric electric circuit (GEC), energetic radiation and electrification of the atmosphere, aerosol and cloud microphysics, thunderstorms, lightning physics, high-energy processes, as well as relations to solarterrestrial phenomena and the evolution of the Earth's climate and atmospheric chemistry. Studies in many areas of atmospheric electricity are rapidly advanced by ground-based, satellite and airborne measurements, laboratory investigations at various scales and chemistry-climate modelling.

Solar-terrestrial influences are explored in several papers. Chum et al. examined the influence of the solar wind on secondary cosmic rays and atmospheric electricity. A relationship between the heliospheric magnetic field, atmospheric electric field, lightning activity, and secondary cosmic rays is investigated with a focus on variations related to the solar rotation. It was found that fluctuations of lightning activity are in phase and in antiphase with the $\mathrm{B}_{x}$ and $\mathrm{B}_{y}$ components of the heliospheric/ interplanetary magnetic field, respectively, in agreement with previous studies. On the other hand, the $\sim 27$-day solar rotation was not significant in the atmospheric electric field measured in Slovakia and Czechia and therefore, no significant influence of $\mathrm{B}_{x}$ and $\mathrm{B}_{y}$ on the atmospheric electric field was observed at these middle-latitude stations. Pilipenko et al. used GLOCAEM data and modeling to address the long-standing problem of the coupling between space weather disturbances and atmospheric electricity. The model predicts that the excitation rate of the E-mode by magnetospheric disturbances is low, such that only a weak $\mathrm{E}_{z}$ response with a magnitude of $\sim$ several V/m will be produced by $\sim 100 \mathrm{nT}$ geomagnetic disturbances. However, at heights $\sim 30 \mathrm{~km}$, the electric field of the E-mode becomes dominant. Bozóki et al. examine the solar cycle modulation of the Earth-Ionosphere Cavity resonator, which encloses electromagnetic radiation of lightning continuing current $<100 \mathrm{~Hz}$, a phenomenon known as Schumann resonances. It is shown that to explain all observations, the effect of solar X-rays and energetic electron precipitation need to be considered, as they modify the quality factor of the cavity mainly over high latitudes.

Kuo et al. verified experimentally the ISUAL array photometer (AP) measured $\mathrm{N}_{2}$ emission ratio $2 \mathrm{P} / 1 \mathrm{P}$ and compared it with the theoretically predicted sprite emission ratio using numerical results on sprite streamers. AP-measured ratios in sprite halo events are consistent with predicted ratios for streamer head electric fields of $3.7 \mathrm{E}_{k}$ and $4.6 \mathrm{E}_{k}$, where $\mathrm{E}_{k}$ is breakdown electric field. Most carrot sprite events initiated at altitudes $67.4 \pm 7.6 \mathrm{~km}$ with smaller estimated electric fields $1 \sim 4 \mathrm{E}_{k}$. Below $60 \mathrm{~km}$, AP-measured ratios fell below the predicted ratio $\sim 1 \mathrm{E}_{k}$.

Disturbances to the atmospheric electric field due to large volcanic eruptions, nuclear accidents, nuclear weapons tests, and the presence of desert dust in the atmosphere is examined in three papers. Xie et al. use an aerosol coupled chemistry-climate model to develop a new global electric circuit model. Their results show that due to the Brewer-Dobson circulation, there are significant seasonal variations in the ion loss due to variations in the volcanic aerosol layer. In the winter hemisphere at 
high latitudes, the column resistance will be larger than the column resistance in the summer hemisphere. With a volcanic aerosol layer in the decreasing phase of solar activity, the column resistance would be more sensitive to fluctuations of the electron flux of precipitating electrons from low to medium energies. Coupling this model with a global circuit sub-model including accurate ion pair production by relativistic electron precipitation could clarify the link between space weather and the troposphere. Kubicki et al. analyze atmospheric electricity parameters measured at the Geophysical Observatory in Świder, Poland, during major events that resulted in the release of a significant amount of artificial radioactive substances in the Earth's atmosphere. Namely, nuclear weapons testing 1958-1965, the Chernobyl disaster in 1986 and the Fukushima accident in 2011. Mallios et al. studied the attachment of ions to settling spherical dust particles iusing a $1 \mathrm{D}$ numerical model that estimates the acquired electrical charge on dust particles and calculates the electrical force that is applied to them. Using observed dust size distributions, the authors find that the particles acquire charge in the range of 1-1,000 elementary charges depending on their size and number density. The particles become mainly negatively charged, but under certain conditions particles $>100 \mu \mathrm{m}$ can be positive. The large-scale electric field can increase up to 20 times when compared to average fair-weather values. The results show that the electrical force is not enough to significantly influence the gravitational settling of the particles. This indicates that the process of ion attachment alone is not sufficient to modify particle dynamics and points to the need to include triboelectric effects and updrafts to fully represent the impact of electricity on particle dynamics in the model.

Hunting et al. assess theoretically how trees alter their surrounding electric fields and empirically compare the influence of trees on the dynamics of the atmospheric potential gradient, positive ions the ground level and electrochemical properties of the soil. It is shown that a substantial increase in atmospheric potential gradient only marginally affects the electric field under the tree canopy, and that soil electrochemical properties are tied to the temporal dynamics of positive ions near the ground level atmosphere. Trees reduce the temporal variability in both, ground level positive ion concentrations and soil redox potential. The results suggest that a tree can alter the temporal variability of the atmospheric electric field at the ground and soil electrochemistry, and it is therefore possible that soil microorganisms, processes and electro-sensitive organisms are indirectly influenced by atmospheric electric fields.

Tacza et al. present potential gradient measurements from five remote stations at high latitudes in the Southern and Northern Hemisphere, to minimize the influence of local effects. They present a first description of new datasets from Halley, Antarctica, and Sodankylä, Finland, along with new criteria for the determination of fair-weather conditions at snow covered sites. It is shown that wind speeds as low as $3 \mathrm{~m} / \mathrm{s}$ can loft snow particles, and that the fetch of the measurement site is an important factor in determining this threshold wind speed. The daily and seasonal analysis of the potential gradient in fair weather conditions shows an excellent agreement with the Carnegie curve of the global electric circuit. This demonstrates that high latitude sites, at which magnetic and solar influences are occasionally present, can also provide globally representative measurements for global electric circuit studies.

Rusz et al. use a large aperture array of microbarometers to calculate the source locations of infrasound emissions from lightning discharges by using the time delays between rapid changes of the electrostatic field and the arrival of the infrasound signals. For most of the analyzed cases, the calculated infrasound source location corresponds to the lightning location determined by the European lightning detection network EUCLID. The method allows for the calculation of the height of the infrasound source, which is typically found $\sim 3-5 \mathrm{~km}$. Tritakis et al. created artificial disturbances such as rifle firings, car engine operation, car radio, and apparatus shaking, near ELF recording stations to identify the impact of such radio frequency interference on the recordings of electromagnetic waves in the Schumann resonance band $<100 \mathrm{~Hz}$. Such disturbances simulate anthropogenic noises from hunters, hikers and campers, which may occur near remote ELF recording stations. The work can assist the differentiation between artificial signals created from anthropogenic activity and natural signals attributed to geophysical phenomena.

\section{AUTHOR CONTRIBUTIONS}

IM conceptualized the idea of the Research Topic "Atmospheric Electricity." IM, MF, KK, and EM are contributors and editors of the Research Topic "Atmospheric Electricity." KK wrote the first draft of this editorial with editing and additional contributions from IM, MF, and EM. All authors listed have made a substantial, direct, and intellectual contribution to the work and approved it for publication.

\section{FUNDING}

IM's work in the SPbU "Ozone Layer and Upper Atmosphere Research Laboratory" was supported by the Ministry of Science and Higher Education of the Russian Federation under agreement contract no. 075-15-2021-583. The work of MF was sponsored by the Royal Society (UK) grant NMG/R1/180252 and the Natural Environment Research Council (UK) under grants $\mathrm{NE} / \mathrm{L} 012669 / 1$ and NE/H024921/1. MF's project work has received funding from the European Union's Horizon 2020 research and innovation programme under the Marie Sklodowska-Curie grant agreement 722337.

\section{ACKNOWLEDGMENTS}

We thank authors of the papers published in the Research Topic "Atmospheric Electricity" for their valuable contributions and the referees for their important reviews. KK, IM, and EM 
acknowledge COST Action CA15211 "ELECTRONET". IM acknowledges Dr. Eugene Rozanov for his help with the organization of the Research Topic "Atmospheric Electricity." This Research Topic has been realized in collaboration with Dr. Svetlana Dementyeva. IM acknowledges the support from Roshan Patel of the "Frontiers in Earth Science" editorial office during the editorial process.

Conflict of Interest: The authors declare that the research was conducted in the absence of any commercial or financial relationships that could be construed as a potential conflict of interest.
Publisher's Note: All claims expressed in this article are solely those of the authors and do not necessarily represent those of their affiliated organizations, or those of the publisher, the editors and the reviewers. Any product that may be evaluated in this article, or claim that may be made by its manufacturer, is not guaranteed or endorsed by the publisher.

Copyright $\odot 2022$ Mironova, Füllekrug, Kourtidis and Mareev. This is an openaccess article distributed under the terms of the Creative Commons Attribution License (CC BY). The use, distribution or reproduction in other forums is permitted, provided the original author(s) and the copyright owner(s) are credited and that the original publication in this journal is cited, in accordance with accepted academic practice. No use, distribution or reproduction is permitted which does not comply with these terms. 